FORMATION Formation emploi

Revue française de sciences sociales

135 | Juillet-Septembre 2016

L'économie verte : rupture ou adaptation de la

formation et de l'emploi ?

\title{
Les Conseillers info énergie : petites mains incertaines des politiques énergétiques françaises
}

The job of "Conseiller info énergie ": small uncertain hands of the French energy policies

Beruf „Conseiller info-énergie“ (Energieberater) : kleine Helfer der französischen Energiepolitik auf unsicherem Posten

Los asesores info-energía : pequeñas manos inciertas de las políticas energéticas francesas

Joseph Cacciari

\section{OpenEdition}

Journals

Édition électronique

URL : http://journals.openedition.org/formationemploi/4830

DOI : $10.4000 /$ formationemploi.4830

ISSN : 2107-0946

Éditeur

La Documentation française

Édition imprimée

Date de publication : 12 octobre 2016

Pagination : 137-154

ISSN : 0759-6340

Référence électronique

Joseph Cacciari, «Les Conseillers info énergie : petites mains incertaines des politiques énergétiques françaises », Formation emploi [En ligne], 135 | Juillet-Septembre 2016, mis en ligne le 12 octobre 2018, consulté le 30 octobre 2020. URL : http://journals.openedition.org/formationemploi/4830 ; DOI : https://doi.org/10.4000/formationemploi.4830 


\section{Les Conseillers info énergie : petites mains incertaines des politiques énergétiques françaises}

JOSEPH CACCIARI

Doctorant en sociologie au sein du Laboratoire méditerranéen de sociologie (Aix-Marseille Université, CNRS, LAMES UMR 7305). Ses thèmes de recherche portent sur la sociologie des groupes professionnels, la stratification sociale et l'économique de la consommation

Résumé

Les conseillers info énergie : petites mains incertaines des politiques énergétiques françaises

Cet article examine la diffusion de l' « économie verte " à partir du métier de Conseillers info énergie (CIE), créé en 2002. Ces conseillers orientent les particuliers dans leur projet d'achat d'équipements d'énergie renouvelable ou de rénovation énergétique de leur logement, afin de favoriser le "verdissement " du secteur de l'habitat. À partir de l'histoire et du positionnement de ce métier, nous soutiendrons que l'économie verte se trouve dans une incertitude vis-à-vis de son institutionnalisation.

Mots clés : métiers du conseil, écologie, habitat, profession

Abstract

The job of « Conseiller info énergie »: small uncertain hands of the French energy policies

This article examines the diffusion of the "green economy" from the Conseiller info énergie job, created in 2002. These advisors direct individuals in their proposed purchase of renewable energy equipment or energy renovation of their homes, to promote the "greening" of the housing sector. From the story and position of this activity, we support the green economy is caught in uncertainty as to its effective institutionalization.

Keywords: Keywords: occupation in advice counselling, ecology, housing, occupation Journal of Economic Literature: J 24, Q 56

Traduction : Auteur. 
On peut saisir les vicissitudes d'une " économie verte " à travers la constitution d'un métier tel que celui de "Conseiller info énergie " (CIE). Cette appellation désigne un groupe de travailleurs qui exercent, auprès des particuliers, une activité de diffusion d'informations en matière de consommation d'énergie et de rénovation énergétique du logement individuel. Les CIE travaillent dans un réseau de lieux d'accueil, les Espaces info énergie (EIE), créés par l'Agence de l'environnement et de la maîtrise de l'énergie (ADEME) et portés soit par le milieu associatif, soit par les collectivités territoriales. L'ADEME est à l'origine de la création de l'appellation et assure le financement des missions des CIE.

L'argumentaire de l'article s'appuie sur les acquis récents de la sociologie des groupes professionnels (Demazière, Gadéa, 2009 ; Champy, 2009 : Vezinat 2010). La notion de groupe professionnel désigne " des ensembles de travailleurs exerçant une activité ayant le même nom ". Ces travailleurs "ne bénéficient pas nécessairement d'une reconnaissance de fait, largement partagée et symbolisée par leur nom, qui les différencie des autres activités professionnelles. En l'absence de réglementation et de codifications formelles, les groupes professionnels sont des ensembles flous, soumis à des changements continus, caractérisés à la fois par des contours évolutifs et une hétérogénéité interne" (Demazière, Gadéa, 2009, p. 20).

Dans cette perspective, la distinction entre " profession » ou " métier » n'est plus complètement pertinente, car les deux termes désignent une position dans le système productif. En faisant notre cette position, nous emploierons indifféremment le terme de «métier» ou de " groupe professionnel " pour désigner les CIE.

Ce courant de recherche de la sociologie des groupes professionnels établit également que la compréhension de l'apparition d'une nouvelle qualification passe par l'examen de sa constitution dans des contextes sociaux mouvants. Ainsi, l'analyse s'intéresse également à la manière dont un groupe professionnel se positionne au sein d'une concurrence interprofessionnelle, pour un territoire d'intervention, par exemple grâce au concept de mandat ${ }^{1}$.

En outre, dans cette approche, il est également habituel de restituer les dynamiques sociodémographiques qui président à la morphologie sociale d'un groupe professionnel ; par exemple à travers la notion de carrière, entendue ici comme la succession des positions occupées par un individu au sein d'une même profession, dans le temps (Becker, 1985 ; Hughes, 1996), et la comparaison des carrières de ses membres. Cette approche montre aussi que "la naissance" (souvent par recomposition d'autres acti-

1. Le mandat renvoie à la revendication du monopole moral, légal et intellectuel sur un domaine d'activité. Comme le précise Hughes : "Non seulement [les membres d'une profession], parce quils sont entrés dans le cercle magique des collègues, ont individuellement licence de faire ce que d'autres ne font pas, mais, en tant que groupe, ils prétendent indiquer à la société ce qui, dans tel domaine de l'existence, est bon et juste pour l'individu et la société» (1996, p. 100) 
vités) et les transformations, voire la disparition d'un groupe professionnel informent autant sur celui-ci que sur le monde social dans lequel il s'inscrit et sur les luttes qui s'y déroulent; notamment celles qui visent la qualification de la réalité sociale, comme lorsqu'il s'agit, dans le cas étudié, de verdir l'économie.

\section{Encadré 1. Méthodologie de l'enquête}

L'article est issu d'une monographie du « métier » de Conseiller info énergie (CIE ) , réalisée entre 2013 et 2015, appuyée sur quatre types de matériaux.

Premièrement, une enquête par questionnaire auprès des $500 \mathrm{CIE}$ exerçant, en France, au moment de l'enquête. Le taux de répondants est de $39 \%$. Malgré ce bon score relatif, il est impossible de se prononcer de façon tranchée sur la représentativité de l'enquête. Néanmoins, le croisement de cette partie de la recherche avec les autres matériaux suggère que les résultats obtenus permettent de caractériser la morphologie sociale du métier (ie. sa structure et son organisation).

Le second type de matériau provient d'entretiens biographiques conduits avec des CIE ou d'anciens CIE de la région d'enquête $(N=16 / 22)$. À partir de ces entretiens, nous avons examiné la structuration locale du métier. Des comparaisons avec les résultats de l'enquête par questionnaire ont aussi permis de rejeter l'hypothèse d'une trop grande spécificité de l'exercice du métier dans la région.

En troisième lieu, l'enquête s'appuie sur des observations du travail de quatre $\mathrm{CIE}$, grâce à une immersion de plusieurs mois au sein de l'association qui les emploie dans une grande ville du sud de la France (200 observations d'entretiens entre CIE et particuliers, soit une centaine d'heures). Ces observations visaient à mettre en exergue le verdissement de l'économie en-train-de-se-faire au guichet des EIE (Espaces info énergie), mais aussi d'examiner l'actualisation de la pratique du conseil en économie d'énergie, d'en approcher la grammaire en situation, par-delà une perception extérieure et intellectualiste de la pratique (produite notamment dans les discours sur la pratique).

Enfin, la documentation officielle et la littérature grise publiées par l'ADEME (Agence de l'environnement et de la maîtrise de l'énergie) ou produites par les CIE eux-mêmes, depuis la création du métier, ont été mobilisées. Il s'agissait essentiellement d'opérer un travail de cadrage de l'activité et des changements éventuels des orientations publiques les concernant.

Ce croisement de matériaux vise la saturation des données par accumulation et croisement des sources et de traces documentaires. On a cherché ainsi une dynamique de changement d'échelle d'observation et de temporalité, autour d'un même objet de recherche, permettant un contrôle plus serré des interprétations et des analyses (Arborio, Fournier, 2010).

Un retour préalable sur la notion de "transition énergétique » est nécessaire. Le " verdissement » de l'économie française gagne ainsi en légitimité, depuis une dizaine d'années, parce qu'un "impératif » de transition énergétique s'impose aux pays occidentaux. Malgré des divergences sur la définition d'une transition énergétique, les options « convergent le plus souvent vers des politiques de sobriété, d'efficacité» (Labussière, Nadaï, 2015, p. 13), avec un usage des énergies non-carbonées qui irait croissant. Les alertes climatiques et l'épuisement des ressources fossiles sur lesquelles s'appuie l'économie occidentale conduisent à envisager un changement de modèle de production et de reproduction de ces sociétés. 
Au-delà du consensus apparent autour de l'impératif de transition énergétique, « sa mise en cuvre n'en fait pas moins l'objet de désaccords» (Ibid., p. 38), au regard des enjeux (changement climatique, inégalités environnementales, désindustrialisation massive, etc.).

L'une des voies possibles consiste à engager une " révolution verte " de l'économie, par exemple en matière de logement, secteur d'intervention des CIE. Si cet "impératif » de transition énergétique marque les champs politique et médiatique, son appropriation par les particuliers reste cependant sujette à interrogation (Comby, Grossetête, 2012).

C'est pour opérer un travail de conversion des ménages à ce " verdissement » de leurs habitudes dans le logement que le groupe professionnel des CIE voit le jour. Plus précisément, le rôle qui lui est conféré par l'ADEME est de favoriser « le passage à l'acte " (dans le langage émique) des particuliers en matière d'économie d'énergie et de rénovation énergétique des logements individuels.

Les CIE sont en quelque sorte les "professionnels de contact" (ou street-level bureaucrats, Liptsky, 1980 ; Neveu, 2015) de la transition énergétique et du verdissement de l'économie dans le logement individuel. En tant que tels, ils leur sont nécessairement antérieurs.

Nous articulons ici la trajectoire de constitution du groupe professionnel des CIE avec les carrières de ceux qui le composent, en envisageant la transition énergétique et le verdissement de l'économie comme processus historiques en-train-de-se-faire à travers eux. Il s'agit en effet ici de souligner une double incertitude qui caractérise les moyens, les finalités et le périmètre d'action des CIE, en faisant l'hypothèse qu'il en va de même pour l'économie verte en général.

Ainsi, nous ferons d'abord l'historique du métier de CIE. Nous présenterons, ensuite, des éléments de cadrage sur sa morphologie sociale : les effectifs, le profil socio-économique des CIE, etc. Enfin, nous décrirons les différentes formes d'engagement dans le métier et le rapport à sa valeur centrale : la neutralité politique et à l'égard du marché dans le domaine de l'énergie (absence de parti pris pour telle ou telle technologie ou de prosélytisme écologique, interdiction de conseiller des opérateurs commerciau, voir partie 2.2). Nous reviendrons ensuite sur la question du mandat défendu par les CIE, pour conclure en généralisant les analyses au processus de verdissement de l'économie française.

\section{La formation du et au métier de CIE : I'impossible production d'une identité sociale?}

Dans cette partie, le fil conducteur va de la naissance du métier de CIE jusqu'à sa morphologie sociale actuelle. Il montre, d'une part, comment se construit et se configure le métier de CIE ; d'autre part, comment il s'inscrit dans un contexte dépassant sa seule émergence et dans lequel l'intérêt politique en faveur du "verdissement » du secteur du bâtiment ou de la «transition énergétique " s’est révélé fluctuant au fil du temps. 


\subsection{Le métier de CIE n'émerge pas sur un terrain vierge}

Le métier de CIE naît, en 2002, sous l'impulsion de l'ADEME, dans le contexte des accords internationaux sur la réduction des gaz à effets de serre et d'une attention politique croissante envers la transition énergétique. Après avoir délaissé le conseil de proximité aux particuliers durant les années 1990, l’Agence y redonne du crédit durant cette période (Poupeau, 2008).

Comme le montre François-Mathieu Poupeau, ce métier n'émerge cependant pas sur un terrain vierge. Il complète le secteur des conseillers en consommation d'énergie, où l'on trouve les conseillers clientèle des opérateurs privés du secteur, les travailleurs sociaux impliqués dans les dispositifs de maîtrise des charges locatives pour les particuliers en difficulté, les techniciens du bâtiment, les vendeurs des supermarchés de bricolage ou d'autres activités de conseillers en énergie plus ou moins proches du secteur public (Akrich, Rabeharisoa, 1989 ; Poupeau, Ibid.).

Dans cet espace professionnel, le métier de CIE est proche d'une logique de service public, aspect qui, comme nous le verrons plus loin, est mal identifié par les particuliers (Pautard, 2012).

Au début des années 2000, le métier de CIE commence à se constituer à travers sa dénomination actuelle et un réseau de lieux d'accueil du public où travailleront désormais les conseillers de l'ADEME : les Espaces info énergie (EIE), vitrines publiques de l'Agence. Ces espaces sont portés par des associations ou des collectivités territoriales et font généralement l'objet d'un cofinancement avec l'ADEME. Il existait 506 CIE en exercice, au $1^{\text {er }}$ janvier 2014, répartis au sein de 262 EIE, dont 20 dans la région de l'enquête (données : ADEME).

\subsection{Le métier de CIE se caractérise par trois périodes}

Les travaux des sociologues François-Mathieu Poupeau (Ibid.) et Éric Pautard permettent de repérer trois périodes du développement du métier de CIE.

La première s'étend de 2001 à 2006. Le réseau EIE se diffuse alors principalement au gré des volontés politiques locales, des engagements militants d'associations qui se saisissent de l'Espace info énergie comme d'une opportunité de valorisation (Pautard, op. cit.).

À partir de 2006, et jusqu'en 2010, le métier connaît une amorce d'institutionnalisation et une plus grande visibilité, avec l'élargissement du réseau et un nombre croissant de contacts avec les ménages. Comme le suggèrent notre enquête par questionnaire et la documentation disponible à l'ADEME, les effectifs de CIE progressent alors d'environ $30 \%$ sur la période. En 2006, l'Agence crée d'ailleurs une nouvelle formation interne destinée à cadrer l'activité des CIE : Devenir CIE. Cette formation devient obligatoire pour tout entrant dans le métier, nous y reviendrons. Durant cette période, les CIE bénéficient également des 
retombées du Grenelle de l'environnement ${ }^{2}$. Le réseau, qui a déjà essaimé sur le territoire, permet à l'État français de déployer une politique d'incitation financière à l'achat d'équipement "vert ", par exemple pour la production d'énergie renouvelable au domicile. La prise de contact avec un EIE permet ici aux particuliers d'obtenir des informations sur ces incitations, mais aussi de voir d'une certaine manière valider leur projet d'achat (il en va de même de la rénovation thermique du logement).

Comme l'indiquent la plupart des enquêtés, mais aussi nos observations et comptages, cette période prend fin avec un retournement de conjoncture en 2011. Le nombre de contacts réalisés par les CIE décline, remettant en partie en cause le réseau EIE et les emplois de CIE associés. On ne peut objectiver cette séquence ; toutefois, c'est a minima le ressenti des CIE enquêtés par entretien.

En 2013, peut-être à la faveur du renouvellement politique national et du retour de la transition énergétique comme cause publique, un Plan quinquennal de rénovation énergétique de l'habitat (ou PREH) est voté. Les EIE deviennent à nouveau un guichet des subventions destinées aux particuliers, et les contacts avec les ménages repartent à la hausse. Au sein de l'EIE observé, cette augmentation est de l'ordre de $60 \%$ pour la fin d'année 2013 (données personnelles construites à partir des fichiers internes à cet EIE). Ce nouvel essor des contacts entraîne notamment des recrutements d'agents afin de faire face à l'afflux d'appels téléphoniques, ce qui sera le cas sur notre terrain d'observation et dans d'autres EIE de la région d'enquête.

\subsection{Emploi et formation des $\mathrm{CIE}$, entre fragmentation et encadrement}

L'histoire du métier de CIE peut être saisie grâce à d'autres indicateurs, par exemple ceux liés aux conditions d'emploi et de formation des CIE, en commençant par la morphologie sociale du métier.

Les recrutements de CIE au sein des EIE sont financés par l'ADEME. Ils visent un nombre de contacts avec les particuliers à atteindre, variable selon la taille des localités où se situe l'EIE (entre 400-600 contacts annuels par conseiller, selon l'ADEME).

Financeur, l'ADEME impose aussi le contenu formel du travail et ses directions, notamment à la faveur de (quasi) notes de service.

À partir de notre enquête par questionnaire, on peut construire un profil de la composition sociodémographique du métier. Ainsi, $80 \%{ }^{3}$ des CIE interrogés sont employés par des associations, habituellement implantées dans des villes moyennes (entre 3000 et 100000 habitants) ou des grandes villes (plus de 100000 habitants). Parmi eux, 80,6 \% ont un

2. Il s'agit d'une séquence politique qui s'étend de 2007 à 2012, et qui visait à préfigurer des changements législatifs en matière d'environnement. Deux lois en naîtront, désignées sous le titre de Grenelle I (2009), qui est une loi de programmation, et Grenelle II (2010) qui complète, applique et teritorialise la précédente. 3. Les données qui suivent sont issues de notre enquête par questionnaire. 
contrat à durée déterminée et 86,7 \% travaillent à temps plein. Le salaire moyen est de $1400 €$, avec des variations en fonction du niveau de diplôme, de l'année de recrutement et du type d'employeur. L'effectif enquêté est majoritairement masculin (63,6\%).

En recoupant l'âge et l'année de recrutement, on constate aussi que $50 \%$ des répondants occupent un emploi de CIE depuis deux à cinq ans et que 62,4\% de l'effectif déclare avoir exercé un autre emploi auparavant. Néanmoins, les effectifs les plus jeunes (biologiquement et professionnellement) sont surreprésentés parmi les recrutements les plus récents (moins d'un an).

S’agissant de leurs originales sociales, les CIE sont recrutés essentiellement dans l'espace des professions intermédiaires, des employés ou des techniciens, et plus particulièrement encore au sein de couples socialement homogames (par exemple des couples où les deux conjoints exercent une profession intermédiaire et ont une origine sociale proche ou semblable) de ces régions de l'espace social.

\section{Tableau 1 - Diplômes selon l'ancienneté de recrutement des Conseillers info énergie ayant répondu à l'enquête par questionnaire}

\begin{tabular}{lcccc}
\hline $\begin{array}{c}\text { Durée de } \\
\text { l'exercice du } \\
\text { métier }\end{array}$ & $\begin{array}{c}\text { Baccalauréat ou } \\
\text { inférieur }\end{array}$ & $\begin{array}{c}\text { Diplôme de 1er cycle universitaire, } \\
\text { BTS, diplôme sanitaire ou social }\end{array}$ & $\begin{array}{c}\text { Diplôme de 2e ou 3e } \\
\text { cycle universitaire }\end{array}$ & Total \\
\hline $\begin{array}{l}\text { Inférieur ou égal } \\
\text { à un an }\end{array}$ & 0 & 61,5 & 38,5 & 100 \\
$\begin{array}{l}\text { Entre deux et cinq } \\
\text { ans }\end{array}$ & 1,3 & 47,5 & 51,3 & 100 \\
$\begin{array}{l}\text { Entre six et neuf } \\
\text { ans }\end{array}$ & 0 & 42,1 & 57,9 & 100 \\
Dix ans et plus & 10,5 & 52,6 & 36,8 & 100 \\
\hline Total & 1,8 & 49,1 & 49,1 & 100 \\
\hline
\end{tabular}

Champ : 195 Conseillers info énergie, France.

Note de lecture : Parmi les Conseillers info énergie répondants, qui ont été recrutés dans une période allant de six ou neuf ans au moment de l'enquête (2014), 57,9\% sont titulaires d'un diplôme de $2^{\mathrm{e}}$ ou $3^{\mathrm{e}}$ cycle universitaire.

Sigle : BTS - brevet de technicien supérieur.

Source : Enquête personnelle, 2014.

Dans la plupart des cas observés, le niveau de diplôme exigé est celui de la licence, plus précisément des licences professionnelles techniques liées aux énergies. Comme le détaille le tableau 1, dans les premières années du réseau EIE, les recrutements ont été plus " ouverts» : vers des individus titulaires d'un diplôme de niveau master (recrutés encore aujourd'hui, mais dans une moindre mesure) et vers des personnes moins diplômées, mais ayant suivi certaines formations reconnues alors par l'ADEME, et les employeurs de conseillers 4 .

4. Du type de celles dispensées par l'Association savoyarde pour le développement des énergies renouvelables 
Ces différences de conditions d'emploi, de générations et de niveaux de formation conduisent à s'interroger sur l'homogénéité d'un groupe supposé relayer une même politique publique : celle de la transition énergétique dans le logement individuel. Au travers de la formation Devenir Conseillers info énergie, l'appariement entre des individus aux parcours hétérogènes et une seule et même fonction est supposé être, en partie au moins, réalisé.

Comme le mentionnent les documents internes à l'ADEME, cette formation est obligatoire pour chaque nouvelle recrue. Une formation au métier existait dès 2002. Elle se déroulait sur quelques jours, parfois sur le lieu de travail. Dans sa version de 2006, elle est organisée sur une semaine dans les locaux de l'ADEME', avec trois objectifs : développer les compétences techniques et relationnelles des CIE afin de les préparer à rencontrer le public ; standardiser le conseil et permettre ainsi une meilleure homogénéité ${ }^{6}$ du métier ; faire prendre conscience au CIE qu'il s'engage, volens nolens, à représenter l'ADEME et les institutions publiques. Une lecture du cahier des charges de la formation montre qu'elle est structurée autour de plusieurs domaines de compétence : technique, relationnelle, connaissance institutionnelle, connaissance du réseau. Elle ne donne lieu ni à un examen ni à la délivrance d'un diplôme. Elle est aujourd'hui en passe d'être réformée dans une version dématérialisée. Elle se tiendrait alors à distance via une plateforme informatique (au moins en partie), directement sur le lieu de travail. Cela ne manquerait pas d'individualiser la formation au métier, avec probablement comme conséquence un émiettement encore plus fort de son identité. Au cours de leur " carrière ", les CIE peuvent également s'inscrire à des formations thématiques (par exemple sur les constructions en bois, etc.) facultatives, proposées par l'ADEME. Nos observations montrent qu'il s'agit d'une démarche relativement fréquente (une fois par an en moyenne) et largement prescrite entre CIE. Il existe aussi une autre modalité de formation au métier : celle du " tuilage ». Dans le langage autochtone, le tuilage désigne la formation par les pairs, sur et par le poste de travail :

"C'est pas le cas pour toutes les régions, mais, ici, on avait des réunions mensuelles. Ça a quand même permis de nouer des liens. Les anciens, on a toujours tuilé les autres. [...] Ceux de l'est de la région qui se tuilent ensemble, par exemple. Cela dit, c'est assez valorisant pour nous les anciens, être un peu les porte-parole des EIE et de ce que ça devrait être. [...] souvent, les anciens ont ce rôle de recadrer, il y a un certain professionnalisme. " (Extrait d'entretien : Claude, 48 ans, CIE depuis 20057)

(ou ASDER). Chez les CIE enquêtés, avoir " fait l'ASDER » est d'ailleurs une marque de prestige.

5. Outre cette formation, les nouveaux entrants dans le métier passent également un examen de connaissances techniques et thermiques. La réussite à celui-ci ne conditionne pas le recrutement, qui intervient généralement en amont, mais entraîne l'obligation de suivre un module de formation complémentaire.

6. C'est ce que révèlent nos entretiens avec un responsable de l'ADEME, des responsables de structures employant des CIE, voire certains CIE eux-mêmes.

7. La trajectoire sociale de Claude et son entrée dans le métier sont détaillées dans la seconde partie. 
Le «tuilage " n’est pas propre au métier de CIE. Fabienne Hanique (2014) a montré, par exemple, l'importance du "tuilage» aux guichets de la poste. Si le tuilage est important dans le cas des CIE, ce n'est pas seulement pour apprendre le " tour de main ", le métier ; il constitue aussi une composante de la production et la reproduction de l'identité professionnelle d'un groupe numériquement faible et dispersé sur tout le territoire. Le tuilage n'est pas toujours possible en direct, au contact d'un pair ou des prédécesseurs sur le poste occupé ; dès lors, il peut emprunter la voie de la formation par le réseau CIE au niveau régional, lors de réunions ou via des outils de communication à distance. Mais le tuilage des CIE rencontre aujourd'hui des difficultés, au moins dans la région étudiée. D'abord, d'un point de vue indirect, avec une fréquence moindre des réunions de réseau, passées de mensuelles à semestrielles. Ensuite, par le renouvellement des effectifs. Ainsi, la remise en cause du tuilage, repérable grâce à nos observations du réseau EIE au niveau local, peut conduire à fragmenter non seulement le système de production et de reproduction de l'identité sociale du métier mais aussi sa mémoire, empêchant l'institutionnalisation des valeurs, sa capacité de mobilisation sociale ou de reconnaissance.

\subsection{Une activité " postée » de conseils à destination des particuliers}

Entretiens et observations permettent de bien définir le contenu du travail des CIE d'un point de vue formel et quotidien. Celui-ci consiste, principalement, en des contacts directs avec des particuliers pour la délivrance d'informations relatives à la rénovation thermique du logement individuel (dont l'achat d'équipement) et aux économies d'énergie dans le logement.

Cette activité se déroule dans un bureau d'accueil, un guichet (nouveau nom donné par le PREH). Les informations sont transmises oralement (au téléphone ou en faceà-face), par écrit (par mail essentiellement) et de façon complémentaire en distribuant directement l'un des dépliants publiés par l'ADEME sur ces questions. On peut appeler ce volet de l'activité des CIE le travail « de permanence ».

Deux modalités principales de mise en relation entre CIE et particuliers existent : la voie téléphonique et l'entretien de face-à-face, avec ou sans rendez-vous ${ }^{8}$. Ces rencontres se font quasi exclusivement au sein des Espaces info énergie?

Les particuliers entrent en contact avec les CIE par une démarche volontaire. Cependant, depuis le PREH, ils le font aussi via un numéro d'appel national qui les oriente vers l'Espace info énergie le plus proche de chez eux.

8. Les entretiens ont duré entre 2 minutes et 1 heure 30.

9. Il y a certainement lieu d'ajouter une troisième modalité de mise en relation entre les CIE et les ménages, qui a trait au volet " animation collective " du métier de CIE : stands d'information dans des foires ou lors d'événements nationaux à déclinaison locale de l'ADEME, etc. 
Théoriquement, depuis la mise en place du Plan de rénovation de l'habitat, seuls les ménages avec un revenu fiscal de référence supérieur à $25000 €$ sont orientés vers les EIE, les autres étant dirigés vers les prestataires de l'Agence nationale de l'amélioration de l'habitat (ANAH), chargés d'accueillir les ménages dits " modestes " ${ }^{10}$. Cela n'empêche aucun ménage, quel que soit son niveau de revenu, de solliciter directement l'EIE de son choix, au risque d'être réorienté.

Tous ces contacts et échanges avec les particuliers (nous n'aborderons pas les contacts avec les professionnels du bâtiment, qui restent marginaux) sont enregistrés dans un logiciel : Contact. Celui-ci permet demesurer l'activité et d'éditer annuellement des rapports circonstanciés destinés aux financeurs de la structure. Ce logiciel sert également d'appui pour orienter les entretiens et conserver les éléments nécessaires à la mémoire de l'activité auprès des particuliers : nature de la demande, informations d'état civil sur le demandeur, type de projet de rénovation ou d'achat, historique des rendez-vous, etc.

Ce panorama du métier de CIE conduit à deux constats. Premièrement, le métier est constitué dans le but d'occuper une position particulière dans le champ du conseil en énergie, peut-être jusqu'alors négligé par l'État, celle d'un pôle public du conseil en énergie et en rénovation thermique, venant compléter d'autres modalités de gouvernement des comportements en la matière (normes techniques du bâtiment, encadrement des prix, etc.). Deuxièmement, on peut déceler la volonté de l'ADEME de réguler le métier par une formation et un cadrage à distance de l'activité, avec peut-être l'objectif de standardiser la profession au cœur des processus de mise en politique de la transition énergétique et du verdissement de l'économie française.

La morphologie sociale du métier témoigne, dans une perspective autant diachronique que synchronique, de la difficulté que peut rencontrer cette volonté de régulation face à l'hétérogénéité sociale de l'effectif, à la variabilité des contextes de travail et des conditions d'emploi, à la remise en cause du "tuilage ». Le métier se trouve probablement dans une situation d'incertitude, de fragilité, quant à ses contours et son identité sociale. La partie suivante va examiner cette hypothèse au plus près des acteurs.

10. Ce dispositif de tri des publics est aussi appelé « Guichet unique de la rénovation " (GUR) ou « Point rénovation info service» (PRIS). 


\section{Un rapport différencié aux valeurs du métier, un mandat malmené}

Ainsi, l'approche formelle du métier de CIE, adoptée dans la partie précédente, met en exergue l'assise incertaine du métier, plus de dix ans après sa création. Dans cette partie, nous allons examiner cette fragilité directement auprès des acteurs, afin d'en mieux saisir les principes générateurs et ses liens avec le contexte de transition énergétique et de verdissement de l'économie française.

\subsection{Des trajectoires d'engagement dans le métier qui configurent le rapport au groupe}

On entrevoit ainsi l'existence de différentes générations de CIE, dans un contexte de relative uniformisation et standardisation du métier. Cette stratification interne rend possible l'existence de formes "d'engagement » différenciées vis-à-vis de l'activité et des valeurs censées être portées par le métier. En effet, chez les CIE, comme chez d'autres groupes professionnels (Cartier, 2003 ; Fournier, 2008), les valeurs et les formes d'engagement dans l'activité sont corrélées à plusieurs dimensions. On constate en effet des "présents décalés" (Fournier, 2008) au sein du groupe et des appréciations différenciées de ce qu'est le métier. Ces décalages de perception signifient que plusieurs définitions du métier peuvent coexister, au même moment, dans un collectif de travail, mais encore que les positions dans le cycle de la carrière sont en décalage. Il y a là une source potentielle de différends professionnels et de déstabilisation du groupe.

Nous allons examiner les conséquences de ces différenciations. Mais d'abord, comment ces formes d'engagement au sein des groupes professionnels se différencientelles ? La littérature (Demazière, Gadéa, op. cit.; Vezinat, op. cit.) repère trois processus pouvant se recouper. En premier lieu, ces formes d'engagement et de rapport aux valeurs du métier ou de la profession sont indissociables des modes de recrutement et de l'expérience vécue par ses membres. Elles ont donc aussi un lien avec l'histoire du métier. En second lieu, les formes d'engagement et les valeurs défendues par les agents dépendent de leur trajectoire sociale et, notamment, de leurs expériences professionnelles et scolaires. Enfin, elles sont liées aux capitaux de toutes sortes détenus par les agents, qui vont déterminer leur position par rapport au métier et à son avenir. Ainsi, cette diversité des formes d'engagement souligne l'existence de différentes définitions de l'activité, à un même moment, au sein d'un même groupe professionnel. 


\section{Encadré 2 : Le parcours de Claude}

La carrière de Claude, 48 ans, CIE depuis 2005, met en exergue ce phénomène pour les CIE. Claude n'exerce aujourd'hui son activité qu'à mi-temps, car il a endossé de nouvelles responsabilités au sein de l'association qui l'emploie. Le métier de CIE constitue pour lui une reconversion tardive. Fils d'un médecin spécialisé et d'une professeure d'anglais, après l'obtention de son baccalauréat, il entre dans le monde professionnel. II travaille quinze ans dans le secteur de la musique à l'étranger, comme ingénieur du son. Il gagne alors « très bien » sa vie (jusqu'à $150 €$ par jour), avant l'effondrement de la filière musicale suite à l'arrivée des plateformes de téléchargement de musique, à la fin des années 1990. Il entame alors une première reconversion dans le management, puis revient en France pour raisons familiales, décidant de changer de voie : le "management, ça n'allait pas ». II souhaite faire « d'une passion un métier» en travaillant dans le secteur de l'environnement. En 2002, à 36 ans, il obtient une licence, profitant à ce momentlà de la création de quelques licences mention " énergie renouvelable » (cinq en France à ce moment-là). Une opportunité se présente qui le mène au métier de $\mathrm{CIE}$ au démarrage du réseau dans la région, entre 2005-2006. II me décrit l'ambiance d'ouverture des possibles qui règne à ce moment-là, revient sur l'idée qu'il y avait alors tout à faire pour le métier. Selon lui, la valeur essentielle des $\mathrm{CIE}$, c'est la neutralité.

Comme on le verra plus loin, cette neutralité concerne l'absence de parti pris pour telle ou telle option technologique, l'interdiction de conseiller des opérateurs commerciaux ou encore le fait d'éviter le prosélytisme écologique. Cette neutralité fait contrepoint aux opérateurs commerciaux dans ce secteur. Claude souhaite que le métier reste engagé dans la pédagogie collective et regrette que « les plus jeunes, qui arrivent sans tuilage », donnent parfois une image « infantile » et administrative du CIE, mettant à mal sa «noblesse».

Les dernières lignes du résumé de la carrière de Claude évoquent les tensions qui traversent le métier, tout en incarnant les propriétés de la morphologie sociale du groupe CIE, décrites dans la première partie. Ce résumé illustre par ailleurs, brièvement, la confrontation de différents modes d'engagement et un conflit générationnel qu’il conviendrait d'interpréter non pas comme un affrontement entre « vieux » et " jeunes », entre entrants et établis, mais en termes de classes et de générations sociales.

À partir de cette carrière se dessine ainsi une partie de l'histoire du métier : d'une ouverture des possibles à une sorte de dévalorisation que les derniers recrutements semblent confirmer, du point de vue de Claude. Ce qu'il qualifie de "noblesse " des CIE paraît étroitement associée à la valeur de neutralité et à un mandat de pédagogie technique. Cette noblesse se perdrait en raison de l'arrivée d'une nouvelle génération de CIE. Mais est-ce bien le cas? Pour le savoir, nous allons procéder à l'analyse de l'actualisation de cette neutralité et de ce mandat, formes élémentaires de la noblesse du métier.

\subsection{Une neutralité bien tempérée}

Ces rapports différenciés au groupe CIE, à partir de différents modes d'engagement dans le métier, peuvent ainsi créer des relations contrastées à des valeurs supposées partagées. Malgré ces variations, une valeur semble pourtant défendue plus que d'autres, celle de neutralité. 
Contrairement à ce que suggère Claude, elle est mise en avant par tous les profils d'enquêtés et dans toutes les situations de face-à-face avec le public observé, mais aussi dans les signatures de mails ou les panneaux d'affichage internes.

L’occurrence « neutralité » recouvre en fait quatre réalités du métier. C'est, en premier lieu, le label officiel apposé par l'ADEME sur les EIE : Neutre, gratuit et indépendant. C'est-à-dire l'identité sociale institutionnellement revendiquée, la rapprochant bien de celle du service public. En second lieu, la neutralité renvoie au fait que les CIE ne doivent délivrer aucune information commerciale aux particuliers, par exemple en recommandant tel artisan pour des travaux ou tel fournisseur pour la consommation d'énergie. Néanmoins, cette neutralité est parfois difficile à respecter, et n'est pas sans ambivalence, comme l'indique Claude dans l'extrait suivant :

"On a des astuces. Il y a des entreprises, elles sont sur nos listes, je ne les supporte pas. Mais faut pas se faire attraper. Donc je dis : "faites faire un autre devis " [...] C'est ça toutes les joies de la politique, en sachant jouer sur tous les cas [...] Quand il y a des entreprises comme X, qui a ruiné la filière solaire, bon, je ne peux pas la rayer de la liste. Donc, quand le mec il vient avec le devis de chez eux, je lui dis de faire des devis comparatifs. S'il vient avec plusieurs devis... ben je trouve les arguments, mais objectifs [...] j'en perds un peu ma neutralité, mais j'en dors quand même la nuit."

Ensuite, la neutralité établit une frontière entre ce qu'est le métier de CIE et ce qu'il n'est pas. Isabelle (CIE) note que c'est " la neutralité » qui lui a permis de distinguer le métier de CIE parmi d'autres métiers possibles du conseil en énergie :

"[CIE, fonctionnaire] tu n'as pas besoin de vendre pour avoir ton salaire par mois. Bureau d'étude, jaurais aimé, mais la partie technique, faut vraiment maîtriser [...] donc c'est vrai que CIE, pour les valeurs, la neutralité, ça permettait d'être plus tranquille. C'est un métier qui, en entonnoir [une sorte d'élimination raisonnée d'alternatives possibles jusqu'à réduire le nombre de choix], allait bien. " (29 ans, CIE depuis 5 ans, parents fonctionnaires, titulaire d'un master ; a exercé auparavant comme chargée de mission environnement pour le Conseil régional)

Enfin, la neutralité est censée conférer un supplément de légitimité auprès des particuliers, vis-à-vis des entreprises privées, notamment. Mais les conditions réelles de cette légitimité créent un jeu de dupes entre le public et les CIE, et la neutralité de ces derniers n'est alors, à bien des égards, que "de façade ". Au quotidien, les CIE relaient en fait des normes d'État en matière d'accès aux incitations financières à la rénovation, de critères techniques d'équipement faisant l'objet d'une subvention ou, encore, de consommation d'énergie ; et ce, sans être bien identifiés à l'État par les particuliers qui ne savent bien souvent pas où ils se trouvent lorsqu'ils sont dans un EIE. Une ambiguïté de statut soulignée par Bertrand (CIE) :

"Alors il y a deux choses [...] déjà, c'est le statut du CIE. Je suis financé par l'Etat, par subvention. Donc, même si je suis pas fonctionnaire, c'est l'Etat qui me paye. Mais mon patron, 
c'est celui de la structure porteuse. Je négocie mes congés, mes absences, mes maladies, etc. Mais je rends compte de mon action à l'ADEME. Donc, c'est très ambivalent et très inconfortable. Finalement, tu as plusieurs chefs, qui ne sont pas toujours d'accord. Le statut est pourri. [...] Enfin, c'est encore plus compliqué, je dois rendre des comptes à tous ceux qui financent, la Région, etc. Mais c'est bien à l'ADEME que je rends compte. Mais l'ADEME a des problèmes aussi avec deux ministères de tutelle, etc. Néanmoins, on peut dire que c'est l'ADEME qui me donne des ordres avec les notes de cadrage. " (44 ans, CIE depuis un an au moment de l'enquête, en reconversion professionnelle, formé à l'ASDER, marié, deux enfants)

Relais de l'État, via l'ADEME, les CIE ne peuvent finalement se prévaloir que d'une neutralité bien tempérée autour d'un objectif : travailler sur la technique, mais plus précisément sur le kWh économisé, à partir des prescriptions de leur donneur d'ordres, inscrites dans le script de l'entretien balisé par le logiciel Contact (mentionné dans la première partie). La technique devient donc une composante essentielle de leur pratique et le bastion central de leur mandat.

\subsection{Un mandat professionnel malmené}

"Nous, c'est la technique ", voilà ce qui se répète lors des entretiens personnels avec les CIE, ce qui se constate dans les questionnaires de recherche ou au cours des observations de terrain. Ce mandat technique est revendiqué par les CIE. Il est étroitement associé à l'idée de neutralité pour définir un conseil technicien sans ambition commerciale ou partisane, autrement dit dans l'intérêt de l'usager ou de l'intérêt général. Selon les CIE enquêtés, ce mandat consiste à élever le niveau de connaissances des particuliers en matière de rénovation thermique ou de consommation d'énergie. Les enquêtés soulignent leur participation à cette sorte de pédagogie de la transition énergétique au niveau local sur le thème technique, se distinguant d'autres professions (commerciale, juridique ou sociale). C'est ce que suggère Isabelle, CIE évoquée plus haut :

" [ily] a des gens qui appellent pour les aides financières. Donc, je leur dis qu'on est essentiellement là pour des conseils techniques. [Avant le PREH], je disais au bout d'un moment de venir en entretien. Là où on a le plus d'arguments, c'est sur place. [...] Ce que je fais, maintenant, c'est que jenvoie par mail le guide [des aides financières] et demande avant l'entretien de prendre les plans [du logement]. Et, ensuite, je leur dis que pour les aides, ils voient par rapport au guide [les fascicules d'informations ADEME]. [...] si on fait toutes les aides, ça dure au moins trois quarts d'heure. On devient conseiller financier et on laisse le technique aux artisans. "

Outre la distinction avec d'autres professions et métiers du conseil, ce mandat technique, tel qu'il apparaît dans cet extrait, renvoie aux interactions de face-à-face, durant lesquelles les CIE peuvent aussi argumenter « sur le plan des logements » et mieux « contrôler » leur identité sociale. Ils contrôlent ainsi l'effectivité de leur mandat, face au public, notamment en recentrant la discussion sur les aspects techniques et en faisant montre de leurs compétences en la matière. 
Cependant, l'observation du travail quotidien des CIE vient contredire la prescription formelle de ce mandat technique, sous deux aspects au moins. D'une part, les contacts téléphoniques tendent à supplanter le travail de face-à-face (en moyenne dix appels pour trois rendez-vous en une demi-journée de permanence) ; cela crée un mécontentement chez certains CIE qui soulignent la difficulté à avoir un «vrai » travail technique au-travers de cette modalité. Ce travail téléphonique semble alors renvoyer à une forme de dépréciation et de standardisation du métier. D'aure part, les entretiens en face-à-face se résument souvent davantage à une vérification de la bonne éligibilité aux incitations financières, qu'à un conseil technique.

\section{Encadré 3 : Extrait de carnet de terrain}

L'examen de différents guichets de CIE (conseiller info énergie) révèle l'importance accordée au thème de l'information technique au public. À disposition, de nombreuses brochures d'information sur l'économie d'énergie et la rénovation thermique, qui mettent en scène la vie domestique. Tous ces documents sont produits par l'ADEME (Agence de l'environnement et de la maîtrise de l'énergie). Les documents internes destinés à cadrer l'activité des CIE insistent sur la vulgarisation des thèmes de la consommation d'énergie, de la consommation rationnelle, de l'efficacité énergétique. Mais les kWh économisés, la diffusion d'informations prescriptives (sur le mode du « il faut »), le nombre de contacts réalisés, c'est-à-dire un rapport au client supposé trop consommateur et peu informé (et si ce n'est pas le cas, il est qualifié de " conscient »), semblent primer sur la pédagogie. L'aspect technique, le confort et l'usage de la consommation sont centraux ; cependant, ils sont réduits à une dimension objectivable et présentiste. II existe une importante standardisation pour tous les publics rencontrés $\left(^{*}\right)$.

$\left(^{*}\right)$ : Il existe des variations dans les attitudes des CIE à l'égard du public et, bien entendu, des variations d'approches de celui-ci. Mais elles ont davantage trait à la façon d'aborder l'usager et à la transformation de la posture du CIE lui-même dans l'interaction, qu'au type de conseil relayé. Ces variations ne sont pas niées ici, seulement écartées car éloignées du cœur de l'exposé.

L'enquête par questionnaire confirme cette idée de substitution de l'administratif à la technique. L'analyse des réponses aux questionnaires montre en effet que les CIE mettent largement les conseils de type administratif, c'est-à-dire ceux relatifs aux aides et soutiens à la rénovation, au premier rang des activités de conseil, le conseil technique passant après ; cela est confirmé par nos observations au guichet des CIE.

Dans ce contexte, travailler sur la technique se résume, le plus souvent, à faciliter l'accès à des dossiers de subvention pour la rénovation. Cela permet, à l'échelle de tout le réseau EIE français, de mettre en comptabilité les $\mathrm{kWh}$ économisés théoriquement par le secteur du logement des particuliers, et favoriser ainsi la perception publique d'un "verdissement " de ce secteur. Une situation qui peut se résumer par cette phrase de Bertrand, évoqué plus haut : "Aujourd'hui, on nous dit rénovation et point." (44 ans, CIE depuis un an au moment de l'enquête, en reconversion professionnelle, formé à l'ASDER)

Ainsi, le métier de CIE se fonde sur la valeur de neutralité. Ce positionnement professionnel vise à distinguer les CIE des autres groupes professionnels du conseil en énergie, 
notamment du secteur privé, mais aussi à les positionner comme « nouveaux » ou « différents " par rapport aux métiers plus anciens, pour lesquels cette valeur n'était pas centrale. Mais, en pratique, cette neutralité est apparue bien tempérée, c'est-à-dire qu'elle masque un parti pris technique et décontextualisant.

En outre, le mandat revendiqué par les CIE, celui de la technique, ne semble pas effectif ; en fait, il est essentiellement attaché à l'économie théorique de $\mathrm{kWh}$ réalisée par les particuliers, indexée sur le nombre de dossiers de soutien à la rénovation pris en charge par les CIE.

Finalement, la position de neutralité et le mandat des CIE n'apparaissent pas pleinement réalisables, les conditions de leur accomplissement n'étant que partiellement réunies. Au prisme de ce groupe professionnel, on peut alors s'interroger sur les ambitions réelles du processus de verdissement de l'économie promues par les institutions publiques via l'ADEME. À ce titre, la perte de noblesse du métier, soulignée par Claude (CIE évoqué supra), semble liée aux hésitations politiques plus qu'aux nouvelles générations de CIE. Ces hésitations se lisent au travers de l'histoire de ce métier, l'un des seuls groupes professionnels publics de contact opérant pour la transition énergétique et l'économie verte auprès des particuliers.

\section{Conclusion}

L'étude du cas des CIE permet ainsi d'affirmer qu'il s'agit bien d'un métier émergent de l'économie verte, entendu comme une activité dont l'acte de naissance est récent et trouve sa raison d'être dans des processus tels que ceux de la " transition énergétique » ou du " développement durable».

Mais ce métier ne naît pas sur un territoire vierge. Il prend place au sein d'un espace professionnel préexistant et concurrentiel, depuis longtemps structuré (Akrich, Rabeharisoa, op. cit.). Il vient alors occuper une place a priori disponible depuis l'ouverture du marché de l'énergie à la concurrence et la privatisation des anciennes entreprises publiques du secteur (Électricité de France et Gaz de France) : celle d'un pôle public du conseil en énergie.

Cependant, tant du point de vue de la définition formelle, de la morphologie sociale, que de l'activité, l'identité et le territoire du métier de CIE apparaissent incertains. Cette incertitude a été saisie à travers les carrières contrastées de certains CIE, à l'aune de leurs conditions d'emploi variables, ainsi qu'en observant l'absence d'effectivité du mandat qu'ils revendiquent. Soulignons que le type de structuration affectant le métier de CIE questionne l'ensemble de la dynamique d'émergence de l'" économie verte " ou de la "transition écologique ", au sens ou l'examen de la constitution du métier de CIE révèle le poids des structures et des trajectoires sociales, des carrières, des difficultés à pénétrer des territoires professionnels balisés. 
Cela met en évidence la difficulté à s’imposer d'un métier supposé changer les « catégories de pensée ", alors même que les structures sociales guidant ses catégories se révèlent toujours efficaces ou opérantes, même chez les commanditaires « du changement ».

Affirmer que les CIE se trouvent dans l'incertitude peut signifier, finalement, que la transition énergétique et les transformations de l'économie vers une plus grande attention écologique manquent d'une assise pratique autonome par rapport aux routines établies. L'une des conséquences pour ces deux ambitions sociétales serait une absence de réelle « mise en politique ", pour reprendre la formule de Neveu (op. cit.), c'est-à-dire l'absence d'effectuation pratique, par-delà les rhétoriques institutionnelles et les exhortations au changement.

\section{Bibliographie}

Akrich M., Rabeharisoa V. (1989), Les conseils en économie d'énergie, Paris, CSI, 123 p.

Arborio A.-M., Fournier P. (2010), L'enquête et ses méthodes, l'observation directe, Paris, Armand Colin, 128 p.

Becker H. S. (1985), Outsiders, étude de sociologie de la déviance, Paris, Métailié, 247 p.

Cartier M. (2003), Les facteurs et leurs tournées. Un service public au quotidien, Paris, La Découverte, $236 \mathrm{p}$.

Champy F. (2009), Sociologie des professions, Paris, PUF, 225 p.

Comby J.-B, Grossetête M. (2012), "Se montrer " prévoyant » : une norme sociale diversement appropriée ", Sociologie, n 3, Vol. 3, pp. 251-266.

Demazière D., Gadéa C. (2009), Sociologie des groupes professionnels : acquis récents et nouveaux défis, Paris, La Découverte, 463 p.

Fournier P. (2008), "Le travail des générations : confronter des présents décalés », in Arborio et al., Observer le travail ; Histoire, ethnographie, approches combinées, Paris, La Découverte, pp. 113-129.

Hanique F. (2014), Le sens du travail. Chronique de la modernisation au guichet, Paris, Eres, 429 p.

Hughes E. C. (1996), Le regard sociologique. Essais choisis, Paris, EHESS, 344 p.

Labussière O., Nadaï A. (2015), L'énergie des sciences sociales, Paris, Athéna, 156 p.

Liptsky M. (2010 [1980]), Street-level bureaucracy. Dilemmas of the individual in Public Services, New York, Russel-Sage Foundation, 300 p.

Neveu E. (2015), Sociologie politique des problèmes publics, Paris, Armand Colin, coll. «U», 288 p. 
Pautard É. (2012), « Du rationnement aux Certificats d'économie d'énergie. Quatre décennies de maîtrise de la demande électrique en France et au Royaume-Uni ", Annales historiques de l'électricité, Paris, 10, pp. 43-53.

Poupeau F.-M. (Dir.), (2008), Gouverner sans contraindre. L'Agence locale de l'énergie outil d'une politique énergétique territoriale, Paris, l'Harmattan, 275 p.

Vezinat N. (2010), «Une nouvelle étape dans la sociologie des professions en France. Bilan critique autour des ouvrages de Didier Demazière, Charles Gadéa (2009) et Florent Champy (2009) ", Sociologie, 3/1, pp. 413-420. 